\title{
Duck egg drop syndrome virus: an emerging Tembusu-related flavivirus in China
}

\author{
LIU PeiPei ${ }^{1,4}$, LU Hao $^{3}$, LI Shuang $^{2}$, WU Ying ${ }^{1}$, George Fu GAO ${ }^{1,4,5,6^{*}}$ \& SU JingLiang ${ }^{2 *}$ \\ ${ }^{1}$ CAS Key Laboratory of Pathogenic Microbiology and Immunology, Institute of Microbiology, Chinese Academy of Sciences, Beijing 100101, \\ China; \\ ${ }^{2}$ Key Laboratory of Animal Epidemiology and Zoonosis, Ministry of Agriculture, College of Veterinary Medicine, China Agricultural Univer- \\ sity, Beijing 100193, China; \\ ${ }^{3}$ Bureau of Life Sciences and Biotechnology, Chinese Academy of Sciences, Beijing 100864, China; \\ ${ }^{4}$ University of Chinese Academy of Sciences, Beijing 100049, China; \\ ${ }^{5}$ National Institute for Viral Disease Control and Prevention, Chinese Center for Disease Control and Prevention, Beijing 102206, China; \\ ${ }^{6}$ Research Network of Immunity and Health, Beijing Institutes of Life Science, Chinese Academy of Sciences, Beijing 100101, China
}

Received May 28, 2013; accepted June 15, 2013

\begin{abstract}
Duck egg drop syndrome virus (DEDSV) is a newly emerging pathogenic flavivirus isolated from ducks in China. DEDSV infection mainly results in severe egg drop syndrome in domestic poultry, which leads to huge economic losses. Thus, the discovery of ways and means to combat DEDSV is urgent. Since 2010, a remarkable amount of progress concerning DEDSV research has been achieved. Here, we review current knowledge on the epidemiology, symptomatology, and pathology of DEDSV. A detailed dissection of the viral genome and polyprotein sequences, comparative analysis of viral antigenicity and the corresponding potential immunity against the virus are also summarized. Current findings indicate that DEDSV should be a distinct species from Tembusu virus. Moreover, the adaption of DEDSV in wildlife and its high homology to pathogenic flaviviruses (e.g., West Nile virus, Japanese encephalitis virus, and dengue virus), illustrate its reemergence and potential to become a zoonotic pathogen that should not be overlooked. Detailed insight into the antigenicity and corresponding immunity against the virus is of clear significance for the development of vaccines and antiviral drugs specific for DEDSV.
\end{abstract}

DEDSV, BYDV, pathology, genome, phylogeny, antigenicity, vaccine

Citation: $\quad$ Liu P P, Lu H, Li S, et al. Duck egg drop syndrome virus: an emerging Tembusu-related flavivirus in China. Sci China Life Sci, 2013, 56: 701-710, doi: $10.1007 / \mathrm{s} 11427-013-4515-\mathrm{z}$

Flaviviruses are positive-sense, single-stranded RNA viruses distributed throughout the world. Over 70 different members in genus Flavivirus of Family Flaviviridae have been discovered to date, and more than $50 \%$ of the known flaviviruses are associated with human disease. The most important human pathogens are yellow fever virus (YFV), Dengue virus (DENV), Japanese encephalitis virus (JEV),

*Corresponding author (email: suzhang@cau.edu.cn; gaof@im.ac.cn)
West Nile virus (WNV), and tick-borne encephalitis virus (TBEV), which are arthropod-borne and transmitted to vertebrates by infected mosquitoes or ticks, producing diseases in human beings and animals. Taking into consideration of the vector, vertebrate hosts, antigenic, phylogenetic, and biogeographical characteristics, flaviviruses are divided into three groups: mosquito-borne viruses, tick-borne viruses, and viruses with no known arthropod vector [1].

Many flaviviruses have been isolated from avian species 
or arthropod vectors [2-5]. As a natural reservoir or amplifier host, birds play an important role in the maintenance and transmission of flaviviruses. Indeed, some flaviviruses cause disease in domestic poultry or captive game birds [5-8]. Outbreaks of WNV in domestic goose and duck flocks have been reported in Israel and North America [9]. A variety of feral and captive birds are also known to be susceptible to WNV infection. Israel turkey meningoencephalitis virus (ITV) in the Ntaya virus group infects turkeys, producing non-suppurative meningoencephalitis and myocardial necrosis with severe egg drop in breeder hens [10]. Recently, Bagaza virus (BAGV) infection was identified as the cause of the death of wild partridges and pheasants in Spain [11].

A newly emerging duck egg drop syndrome epidemic has spread in the duck-production provinces in China since 2010. The infected ducks manifest clinical symptoms including anorexia, diarrhea, ataxia, paralysis, and most importantly, a heavy drop in egg production that results in huge economic losses [12-14]. The causative agent of this epidemic is a newly emerging flavivirus named duck egg drop syndrome virus (DEDSV) [15]. The virus is genetically closely related to the Tembusu virus (TMUV) and Sitiawan virus (STWV), but these viruses are dissimilar in disease association and host association, although some current literatures refer this virus as duck TMUV [13,14,16-23].
Complete genome sequence analyses, including phylograms, predicted genomic secondary structure (SS), and conserved sequences or domains in deduced structural and non-structural proteins, have illustrated DEDSV's reemergence and potential threat to human public health in the future [15,20-24]. Thus, the progress that has occurred in characterizing and rapidly detecting DEDSV will be briefly discussed, and antigenic properties inferred from other closely related flaviviruses will also be summarized to fuel specific vaccine, antiviral drug, and therapeutics development.

\section{Clinical and pathological data}

In spring 2010, outbreaks of duck egg drop syndrome characterized by a severe decline of feed uptake and dramatic drop in egg production in laying ducks were reported from a series of farms in southeast China [12]. The disease quickly spread northward, and most farms with layer or breeder ducks were affected by the end of the year. Outbreaks have been reported in Anhui, Fujian, Guangdong, Hebei, Henan, Hubei, Hunan, Jiangsu, Jiangxi, Zhejiang, and Shandong Provinces, Guangxi Zhuang Autonomous Region, Beijing and Shanghai Municipality [12-14,18,20,21,25] (Figure 1).

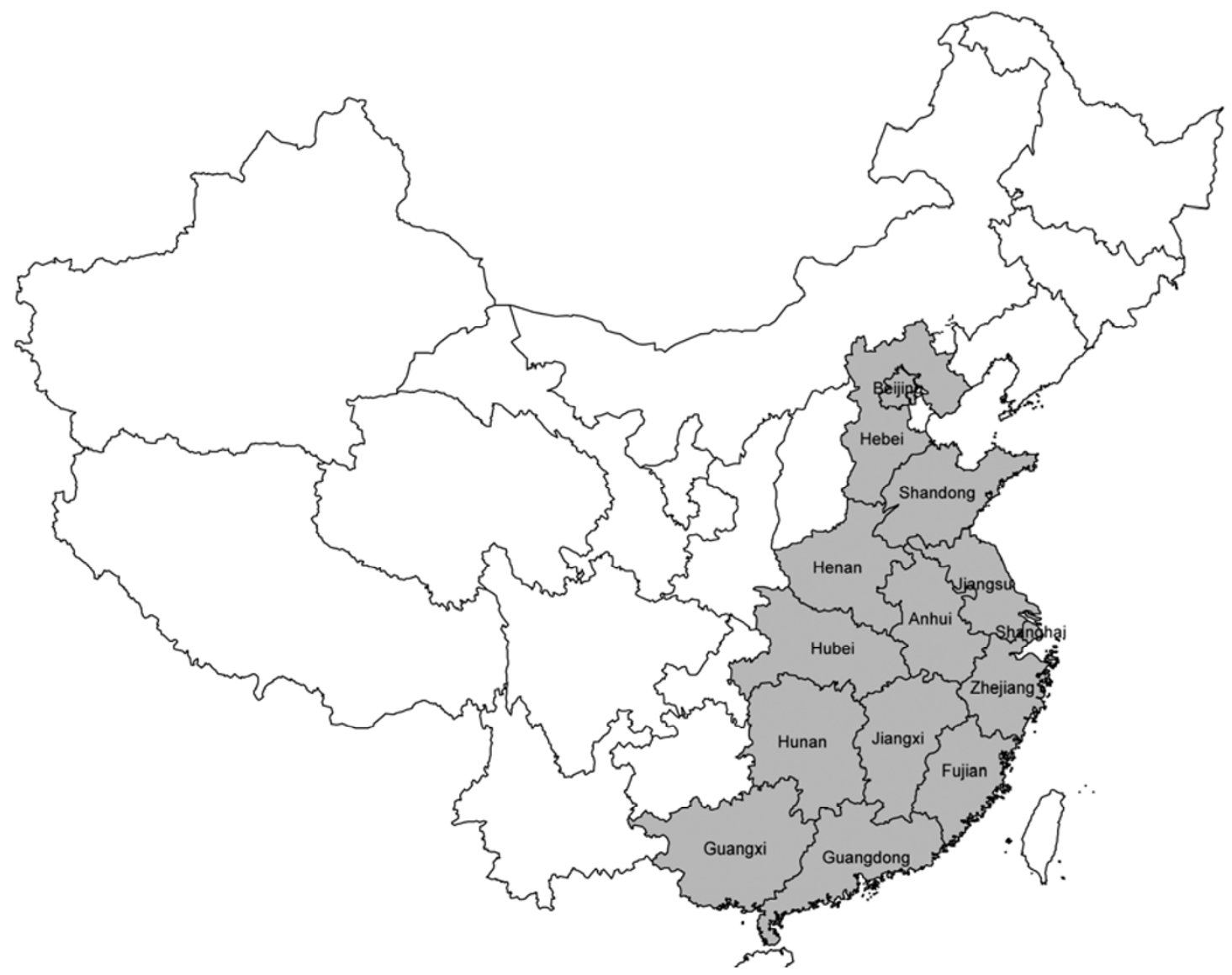

Figure 1 Regions of DEDSV infection outbreaks in China. The provinces, autonomous regions, or municipalities affected are indicated in gray. 
In the field outbreaks, the disease occurs with the greatest incidence in laying ducks (including breeding flocks). The first sign in the infected flock is a decline of feed uptake. This is quickly followed by a decrease in egg production, which drops to lower than $10 \%$ in $10 \mathrm{~d}$. Some affected ducks exhibit neurologic dysfunction characterized by progressive paralysis. Birds are also reluctant or unable to walk. Signs of incoordination are evident when disturbed, and torticollis and opisthotonus can be observed. The mortality ranges from $5 \%-30 \%$ depending on the supportive treatment measures taken by the farmers.

At necropsy, severe ovarian hemorrhage, ovaritis, and regression are consistently found in the affected ducks (Figure 2). Ruptured ovarian follicles and peritonitis are also observed in some affected ducks. Enlarged spleen is occasionally found. Microscopic lesions are mainly found in the ovary with marked hemorrhage, follicle atresia, and rupture. The ruptured follicles and interstitial tissues are filled with round or granular eosinophilic bodies. Additionally, the encephalitis includes focal gliosis, lymphocyte infiltration, and even necrosis in the brain, which are the main pathological changes in most flavivirus infections. Changes in other organs, including nephrosis, pneumorrhagia, and necrosis of the spleen, have been reported [26]. However, non-specific changes could not be ruled out because these lesions were not consistently reproduced by different reports, and no special pathogen-free ducks were used in the experimental infections.

In addition to the DEDSV infection in adult laying ducks, the causative pathogen has been isolated from young meat-type ducks, geese, house sparrows, and a dead racing pigeon [16,20,21,25,27]. The virus-infected ducklings and geese display various degrees of neurological signs, ranging from recumbency to leg and wing paralysis. Affected birds are culled or die from trampling in the field. It is noteworthy that DEDSV has also been detected in chickens with egg drop disease; clinical samples revealed that 138 of 246 (56.1\%) chicken samples were positive for DEDSV [21].

As a newly emerging duck disease, limited experimental data are available for DEDSV transmission thus far. Yan et al. [28] show that high levels of virus can be detected in the tracheas of infected ducks, and the virus can directly transmit to co-habitant birds. The disease characteristics, including sudden outbreak, quick spread, and extremely high morbidity, strongly support direct duck-to-duck transmission. However, transmission by mosquito biting cannot be ruled out because genes from a duck Tembusu-like virus have been detected in mosquitoes trapped at a duck farm during an outbreak in China (personal communication). However, outbreaks of the 2010 infections continued in late autumn and winter in northern China where the mosquitoes are less active, suggesting that mosquito transmission was not the main route.

\section{The virus}

The etiologic agent of duck egg drop syndrome was first isolated from sick ducks by duck embryo inoculation [12]. The virus replicates in both duck and chicken embryos, causing embryonic death. It can be propagated in duck embryo fibroblasts, baby hamster kidney cells (BHK-21), African green monkey kidney cells (Vero), chicken fibroblast (DF-1), and Aedes albopictus cells (C6/36) [12-14,20,25].

Like other flaviviruses, mature DEDSV virions have a nucleocapsid enclosed by a phospholipid bilayer envelope. The surface of the virus particles contains two viral structural proteins, E and M (Figure 3A). Electron microscopy of virus-infected cells revealed viral particles in the cytoplasm, with a diameter of approximately $50 \mathrm{~nm}$ (Figure 3B).

As for all positive-strand RNA viruses, the DEDSV genome is infectious $[22,29]$. To date, complete sequences of

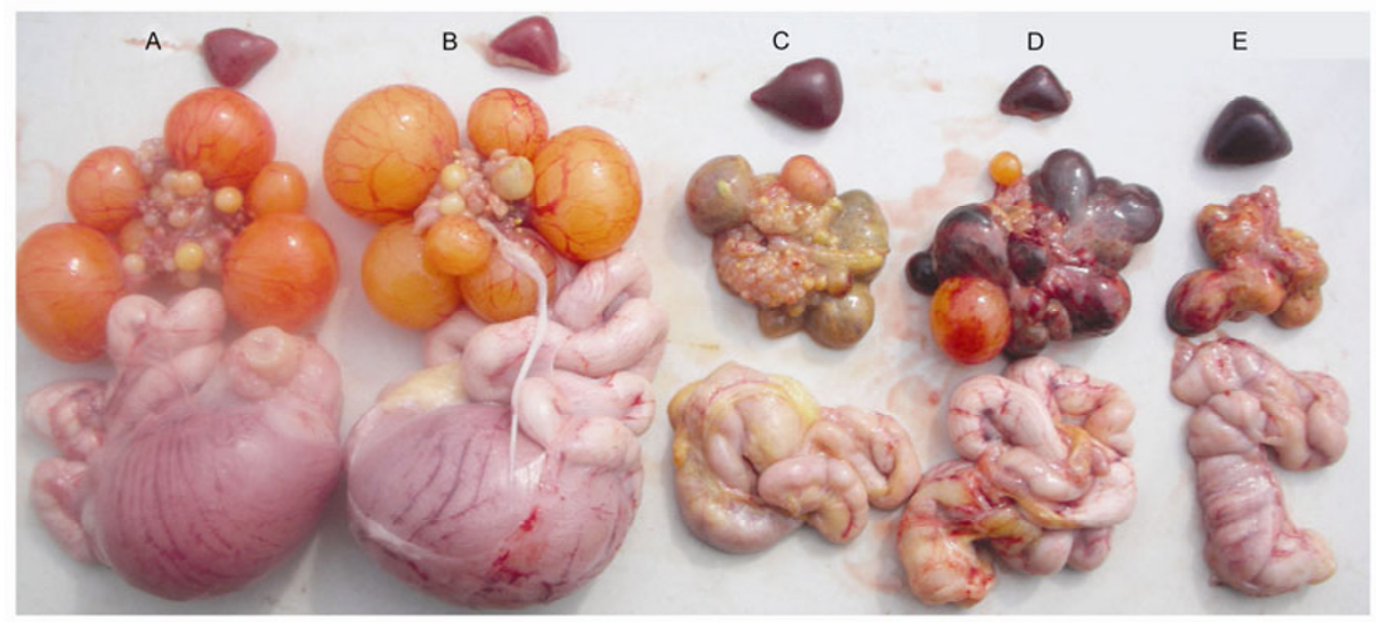

Figure 2 Gross lesions of laying ducks experimentally infected with the DEDSV-JXSP strain. Three ducks were autopsied on day 5 after infection. A and $\mathrm{B}$, The ovaries and spleens of ducks from the non-infected group (control). C-E, Degeneration and severe hemorrhage in ovaries were observed in all infected ducks. 

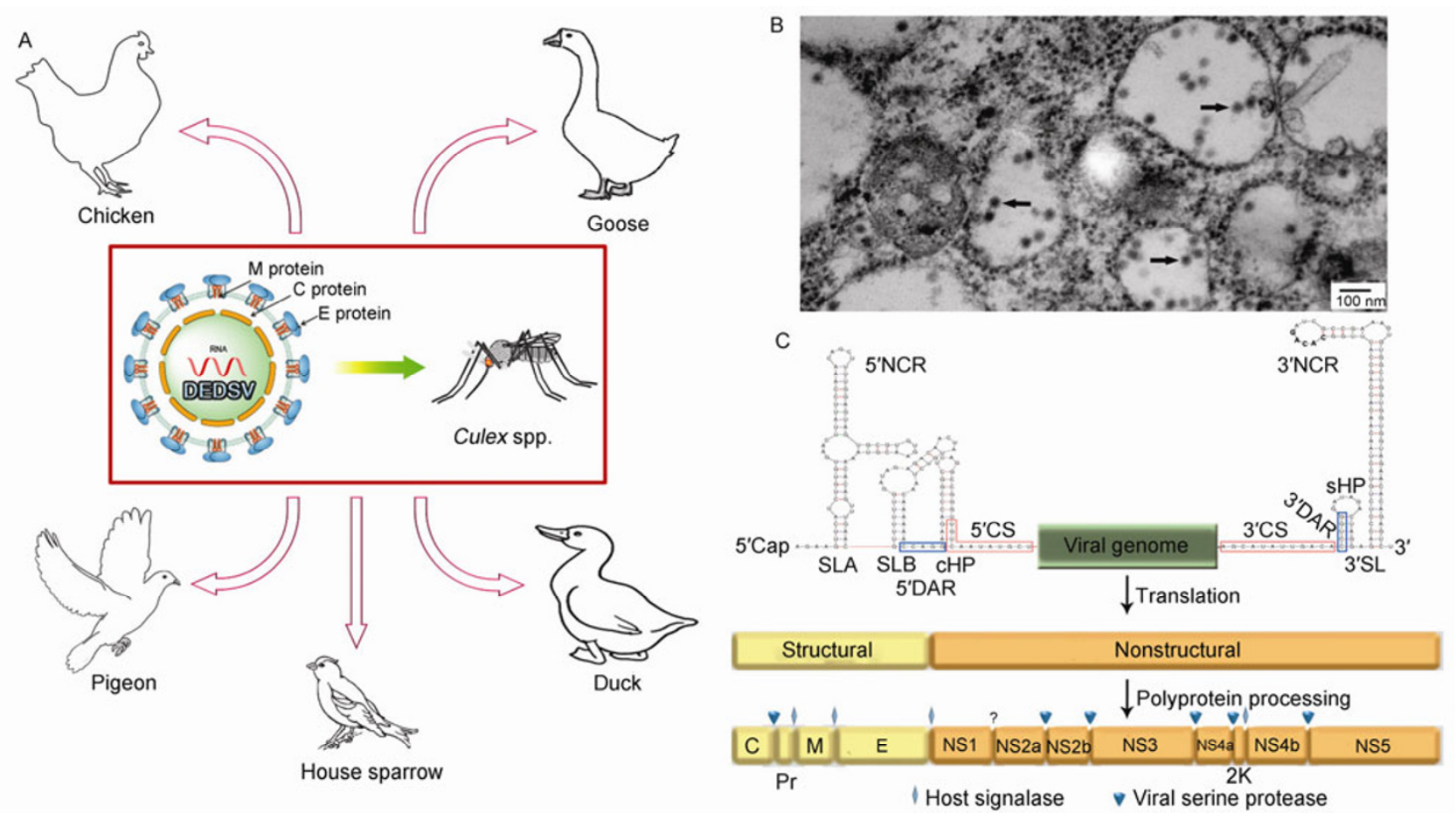

Figure 3 Properties of DEDSV. A, In the red box, the left is a schematic representation of a DEDSV particle. Though not proven, we still cannot rule out Culex spp. as the natural vector of DEDSV (right part in the red box). DEDSV strains have adapted themselves in five vertebrate hosts, as illustrated in the figure. B, Electron micrograph of purified DEDSV. The arrows indicate the DEDSV virions with a diameter approximately 50 nm. C, The upper portion depicts the genomic structures, including the predicted SSs of the $5^{\prime}$ and $3^{\prime}$ terminal non-coding regions. The lower portion illustrates the deduced cleavage sites of the DEDSV polyproteins.

14 DEDSV strains, including two strains isolated from geese, one from a pigeon, and the other 11 from ducks, have been released in GenBank (Table 1). While the genomes of 14 of the 15 strains are of the same length (10990 nt), the strain TA genome is shorter by $4 \mathrm{nt}$ [30]. Thus, we suspect that its genome has not been fully sequenced. As illustrated in Figure 3C, the entire genome of DEDSV is $10990 \mathrm{nt}$ long, has a type 1 cap (m7GpppAmp) structure at the $5^{\prime}$ end, and lacks a poly(A) tail at the $3^{\prime}$ end. The complete ORF, flanked by 5'- and 3'-NCRs of 94 and 618 nt, respectively, is predicted to encode a large polyprotein of 3425 amino acid (aa). After co- and post-translational cleavage by host signalase or viral serine protease, the polyprotein gives rise to 10 viral proteins, including three structural proteins $(\mathrm{C}$, PrM, and E) and seven nonstructural proteins (NS1, NS2a, NS2b, NS3, NS4a, NS4b, and NS5), which is typical of flaviviruses [15-19,25,30].

Phylogenetic analysis based on the nucleotide sequences of $\mathrm{E}$ and NS5 demonstrate that TMUV is most closely related to DEDSV [12-14]. Furthermore, phylograms based on the coding sequences indicate that DEDSV belongs to the Ntaya virus (NTAV) group and is most closely related to TMUV and STWV (Figure 4). Pairwise comparison of the coding sequences of DEDSV with that of TMUV and STWV revealed that they are highly closely related $(97 \%$ and $96.5 \%$ aa identity, respectively) [15]. However, to demarcate a new species, several criteria must be satisfied, e.g., nucleotide and deduced aa sequence data, antigenic characteristics, geographic association, vector association, host association, disease association, and ecological characteristics [31]. Despite the fact that DEDSV is genetically closely related to TMUV, these viruses exhibit distinct disease association and host association. TMUV was first isolated in 1957 from Culex tritaeniorhynchus in Malaysia [32]. Moreover, antibodies against TMUV have been identified in humans [33,34], but to date, no disease is associated with TMUV. With respect to the remaining four species-defining items, additional experimental data are required. Thus, we suggest that the virus should be given a new name, i.e., DEDSV (also called Baiyangdian or BYD virus initially), rather than being referred to as a genotype of TMUV.

Moreover, we found that DEDSV clusters with a group of viruses that diverged from the Japanese encephalitis virus (JEV) serocomplex cluster. Most importantly, these two clades primarily contain Culex spp.-associated viruses, which tend to be ornithophilic and anthropophilic in their host-feeding preference (Figure 4) [35,36]. Furthermore, the phylogenetic cluster that contains DEDSV exclusively contains Old World viruses of either African or Asian origin, diverging from a sister group of New World viruses, i.e., Ilheus virus (ILHV) and Rocio virus (ROCV). In turn, the JEV group viruses segregate from this DEDSV-related cluster, which comprises Old World viruses (with the exception of a single New World virus SLEV that diverged 
Table 1 Summary of the DEDSV strains whose complete genome sequences have been released in GenBank

\begin{tabular}{ccc}
\hline DEDSV strains & Source of isolation & $\begin{array}{c}\text { Location of isolation } \\
\text { (province and municipality in China) }\end{array}$ \\
\hline byd1 & Egg-laying duck & Hebei \\
JXSP & Meat-type duck & Beijing \\
Duan & Egg-laying duck & Beijing \\
JX2 & Egg-laying duck & Jiangxi \\
JS & Egg-laying duck & Jiangsu \\
Goose & Goose & Beijing \\
Pigeon & Pigeon & Beijing \\
ZJ-6 & Pekin duckling & Zhejiang \\
WJ-1 & Muscovy duck & Guangdong \\
YY5 & Shaoxing duck & Zhejiang \\
ZJ-407 & Breeder duck & Zhejiang \\
GH-2 & Goose & Zhejiang \\
JR & White Kaiya duck & Fujian \\
TA & Breeder duck & Shandong \\
\hline
\end{tabular}

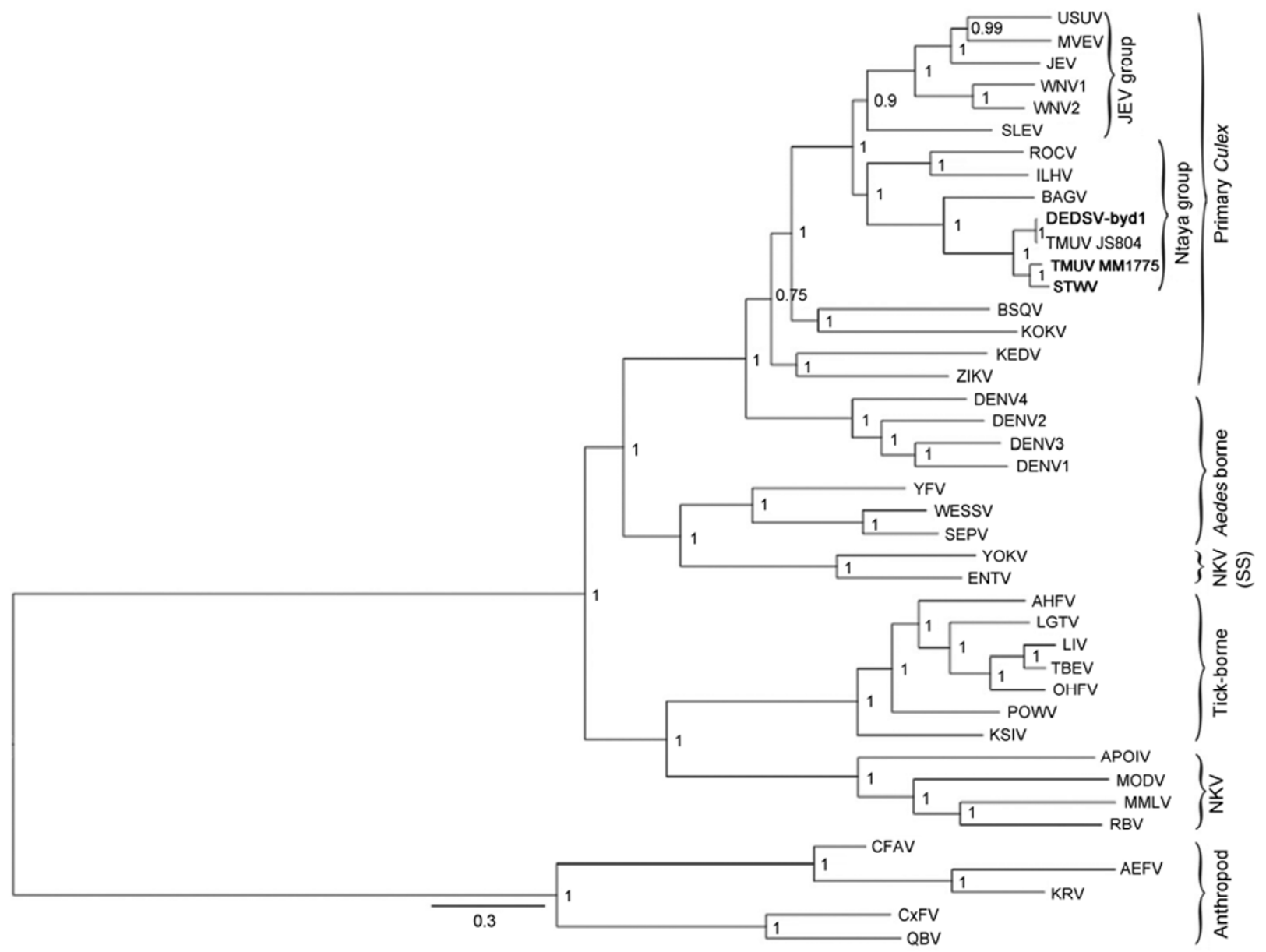

Figure 4 Bayesian phylogeny of representative flaviviruses with DEDSV, TMUV, and STWV. Posterior probabilities are shown for all of the clades. The phylogenetic tree is based on the ORF sequence dataset. DEDSV strain byd1, TMUV strain MM1775, and STWV are highlighted in bold. All horizontal branch lengths are drawn to scale; bar, 0.3 substitutions per site.

from the Old World viruses). Moreover, DEDSV has been isolated from both ducks and domestic birds like geese $[15,17,21]$, chickens [21], and pigeons [15], as well as wild species such as house sparrows [20] in the winter, indicating that bird migration and transport may be a plausible trans- mission route. However, DEDSV infection has been reported to lead to the cytopathic effect in many cell lines, including the mosquito cell line C6/36 [20,25]. Thus, the most likely evolutionary profile for DEDSV, and indeed for TMUV, is that they represent Old World lineages, probably 
of African origin (cf BAGV), that were dispersed into Asia via migratory birds and ornithophilic Culex spp. mosquitoes [15].

Viral RNA genomes not only code for proteins but in many instances also carry RNA motifs that play a crucial role in the viral life-cycle [37]. The 5'- and 3'-ends contain many regulatory elements for viral RNA replication and translation. Moreover, the presence of inverted complementary sequences at the ends of viral RNAs has been observed [38-40]. These elements can mediate long-range RNA-RNA contacts to induce flavivirus genome cyclization, which is a prerequisite for RNA replication [38,39,41,42]. Complementarity, rather than the nucleotide sequence per $s e$, is essential for RNA synthesis [43]. Three crucial sequence motifs complementary between the 5'- and 3 '-ends have been reported for mosquito-borne flaviviruses: the $5^{\prime}-3^{\prime}$ cyclization sequences $\left(5^{\prime}-3^{\prime} \mathrm{CSs}\right), 5^{\prime}-3^{\prime}$ upstream of AUG region (5'-3'UAR) elements, and $5^{\prime}-3^{\prime}$ downstream of AUG region (5'-3'DAR) elements [37,42-48].

The predicted SS at the 5'-end of the flavivirus genome consists of a large stem loop (SLA) with a side loop, which looks like a Y and is thus called a Y-shaped structure [38]. Both in vivo and in vitro data support the idea that the SLA functions as the promoter element for the viral polymerase rather than other elements [45]. A study of DENV replication demonstrates that at least one $\mathrm{U}$ in the bottom bugle of the SLA is required for viral replication [49]. Oligo(U), located downstream of the SLA, functions as a spacer that enhances viral RNA synthesis and is shared by all of the flavivirus genomes [49,50]. In most cases, a second short stem loop (SLB) that contains the 5'UAR sequence is formed and ends at the translational start codon [38,51]. The 5'DAR is located downstream of the start codon. A stable capsid hairpin (cHP) that participates in start codon selection during viral translation [52] and the following 5'CS are also common for mosquito-borne flaviviruses.

For DEDSV, as illustrated in Figure 3C, the SS of the 5'NCR (predicted using the mfold program [53]) revealed that the first domain of approximately $70 \mathrm{nt}$ is predicted to fold into an SLA, which has two bulges, a side stem loop, and a top loop. Furthermore, there is one $U$ residue in the bulge. The following 6-nt Oligo(U) spacer, SLB, 5-nt 5'DAR, and 12-nt 5'CS (Figure 3C) demonstrate their conservation. Furthermore, multiple alignments of all of the 15 strains of DEDSV show that the other 14 strains also possess these features in their $5^{\prime}$ terminal region. Notably, a 5'UAR does not exist in the DEDSV genome.

The $3^{\prime} \mathrm{NCR}$ of the flavivirus genome can be divided into three domains from 5' to 3': Domain I (D1), Domain II (D2), and Domain III (D3). D1 is most variable, while D3 is most conserved. D2 looks like a dumbbell (DB), containing conserved sequences 2 (CS2) and RCS2 (repeated CS2) present in all mosquito-borne flaviviruses [39]. CS2 is proposed to regulate the translation of the viral RNA in Dengue virus type 2 (DENV2) [46]. The cyclization sequence, i.e., CS1, belongs to D3. Downstream of CS1 is a long stem loop (3'SL) shared by all of the flaviviruses. The 3'SL includes both a long stem loop and a short hairpin (sHP) just before the stem loop. The $3^{\prime} \mathrm{CS}$ is located upstream of the sHP, while the $3^{\prime} \mathrm{DAR}$ and $3^{\prime} \mathrm{UAR}$ follows the $3^{\prime} \mathrm{CS}$ in the $5^{\prime}$ to $3^{\prime}$ direction. SS prediction of the $3^{\prime} \mathrm{UCR}$ of DEDSV illustrates that except for the $3^{\prime} \mathrm{UAR}$, all of the common structures exist [15].

Moreover, there are some conserved primary elements, i.e., CS sequences, in the $3^{\prime} \mathrm{NCR}$ of flaviviruses [39]. Compared to other Ntaya group viruses, DEDSV has a CS pattern of CS1-CS2-RCS2-CS3-RCS3 [15,17,19,25], which is also shared by JEV complex viruses. However, BAGV, ILHV, and ROCV are different from DEDSV. CS1 plays a critical role in viral genome cyclization for virus replication, and CS2 likely enhances translation of viral RNA. However, the functions of the other CS sequences are currently unknown. Indeed, they may just be remnants of evolution of these six long repeated sequences [39], though further studies are clearly required to elucidate this point. DEDSV also shares another conserved motif in its $3^{\prime}$ UTR, i.e., the pentanucleotide CACAG in the top loop of the $3^{\prime} \mathrm{SL}$, which is proposed to be critically important for viral RNA replication [54,55].

Most of the DEDSV polyprotein cleavage site analyses coincide with each other, i.e., the first or second amino acid residues directly flanking the protein cleavage sites are mostly conserved among all mosquito-borne flaviviruses, and differences are found in residues not directly flanking the cleavage sites. All sites cleaved by the viral serine protease (Cv/Ci, NS2a/NS2b, NS2b/NS3, NS3/NS4a, NS4a/2K, and NS4b/NS5) and furin (or furin-like protease) (Pr/M) occur after two C-terminal basic residues, typically KR, RR, or QR (Figure 3C). In the sites cleaved by host proteases, the C-terminal and N-terminal aa immediately flanking $\mathrm{M} / \mathrm{E}$, $\mathrm{E} / \mathrm{NS} 1$, and $2 \mathrm{~K} / \mathrm{NS} 4 \mathrm{~b}$ are generally similar to TMUV and other flaviviruses. The NS1/NS2a site cleaved by an unknown cellular protease occurs after $\mathrm{V}-\mathrm{X}$-A residues (in which $\mathrm{X}$ is variable). The lengths of the predicted cleavage proteins are as follows: capsid (C), 120 aa; premembrane (PrM), 167 aa; envelope (E), 501 aa; nonstructural protein 1 (NS1), 352 aa; NS2A, 227 aa; NS2B, 131 aа; NS3, 619 aа; NS4A, 126 aa; 2K, 23 aа; NS4B, 254 aa; and NS5, 905 aа $[15,25]$. However, Liu et al. [30] report a different prediction, where the PrM is longer, and NS1 is shorter (the difference in each is $10 \mathrm{aa}$ ). Another report from Diao's group includes an absolutely distinct prediction, in which most of the cleavage sites are not identical to other predictions [19].

The conserved cysteine residues involved in intramolecular disulfide bonds in PrM, E, and NS1 number 6, 12, and 12 , respectively, which is in accordance with other flaviviruses. However, the number of potential N-linked glycosylation sites in PrM is slightly different, which has been reported by different groups [15-17]. C and E have con- 
vergent predictions of two and three glycosylation sites.

For flaviviruses, three structural proteins and seven non-structural proteins cooperate with each other to facilitate the viral lifecycle. Alignment of the deduced polyproteins of the 15 released DEDSV strains show that E, NS3, $2 \mathrm{~K}$, NS4B, and NS5 have a lower ratio of mutations compared to others (average value $=2.5 \%$ ). $2 \mathrm{~K}$ is an internal signal peptide. The other four proteins all play key roles in viral life.

The dimeric E proteins located on the surface of the viral envelope are generally involved in receptor binding during infection and membrane fusion in the endosome in an aciddependent manner [56]. Each E monomer is composed of three domains with different functions. A highly conserved fusion peptide is located at the tip of Domain II, i.e., aa 98-110, mediating insertion into the target cell membrane. This fusion loop is also shared by DEDSV. Comparison of the DEDSV E protein sequence with that of JEV group viruses shows that the suspected pathogenicity determinant, i.e., the putative conserved integrin binding motif $\operatorname{RGD}(\mathrm{E})$, which is a suspected pathogenicity determinant of flaviviruses [57], is mutated to SGK in DEDSV. In addition, E306 and D389, which are involved in viral attachment to sulfated proteoglycans for JEV group viruses, are mutated to $\mathrm{N}$ and $\mathrm{K}$, respectively. These mutations may account for the different host range and pathogenicity of DEDSV relative to the JEV group viruses.

NS3 is a large multifunctional protein, of which the $\mathrm{N}$-terminal third is a catalytic domain of a trypsin-like serine protease complex, while the C-terminus encodes an RNA helicase. In addition, NS3 contains RNA triphosphatase activity. The proposed conserved catalytic triad and substrate-binding pocket in JEV group viruses also exist in DEDSV in the form of H47-D75-S135 and G133-S135G136-G148-L149-G153, as well as the RNA helicase motif D285-E286-A287-H288 [15].

NS5 is an RNA-dependent RNA polymerase ( $\mathrm{RdRp}$ ). DEDSV also resembles other flaviviruses in the composition of the RdRp catalytic motif, i.e., G667-D668-D669 is also found in the C-terminus of the NS5 protein. The remaining seven proteins play roles in virus infection (e.g., PrM), replication (e.g., NS1), assembly (e.g., C and NS2A), and enzymatic processes (e.g., NS2B). However, no related conserved motifs have been reported to date [58].

\section{Immunity and antigenicity}

Target cells for flaviviruses infection include monocytes, macrophages, and dendritic cells (DCs) [59]. In particular, infection of DCs is important because these intradermal cells can be primary targets early in infection [58]. The virus first attaches to the cell surface and thus enters the cell by receptor-mediated endocytosis [59], in which the E protein plays a dominant role. The $\mathrm{E}$ protein both mediates cell attachment and fusion and is crucial in the generation of neutralizing antibodies and the induction of a protective immune response [60].

Solved structures of the E proteins of JEV [61], WNV [62], DENV [63], and TBEV [64] reveal that E poses a canonical dimeric form, which is consistent with cryoelectron structures of $\mathrm{E}$ in mature virions [58,60]. Furthermore, monomeric $\mathrm{E}$ has three discernable structural domains in common for flaviviruses, which is thought to be due to the six absolutely conserved disulfide bridges [60]. For DEDSV, the 12 conserved cysteines and high homology to the JEV serocomplex indicate that $\mathrm{E}$ protein likely has a similar three-dimensional structure to that of the JEV serocomplex. Thus, we elucidate DEDSV's potential antigenicity by dissecting that of closely related flaviviruses.

For TBEV, on which the most complete antigenic analysis had been performed, the central domain I of E protein contains epitopes that determine distinct subtypes, while two loops in domain II, involved in dimer contacts, correspond to group-reactive epitopes. Furthermore, epitopes in domain III are complex specific [65].

The cross reactivity of DEDSV with several representative flaviviruses demonstrates that DEDSV can cross-react with TMUV, BAGV, DENV-2, TBEV, and JEV complex viruses including JEV and WNV [15]. Although the detailed profile of the epitopes of DEDSV recognized by representative flavivirus-derived antisera is still unclear, we speculate that there must be some existing antisera (antibodies) that can cross neutralize DEDSV, which would be a promising anti-DEDSV therapy while specific anti-DEDSV drugs are still unavailable.

Most pathogenic flaviviruses are associated with neurologic disease [59]. In light of the clinical manifestation and pathology [12-14], we anticipate that DEDSV is also neuroinvasive and neurovirulent. For the neuropathogenic flaviviruses, both innate and adaptive immunity play roles in virus eradication and in immunopathology. The toll-like receptor 3 (TLR3) and retinoic-acid-inducible protein 1 (RIG-1) pathways are designed to sense extracytoplasmic or cytoplasmic structured flavivirus RNA, leading to IFN regulatory factor 3 (IRF-3) and nuclear factor-kappa B (NF- $\kappa$ B) activation and induction of interferon- $\beta[58,60]$.

To combat the immune system, flavivirus nonstructural proteins inhibit IFN-induced signaling through the IFN receptor and the JAK-STAT pathway. Also, these viruses prevent the induction of IFN through activation of IRF-3 [58]. Activated macrophages are capable of producing large quantities of tumor necrosis factor (TNF), nitric oxide (NO), and other soluble mediators that are potentially damaging to neurons [59].

Aside from innate immunity, the organisms also utilize neutralizing antibodies to control viral infection and dissemination. Most of the antibodies are against $\mathrm{E}$ protein, but anti-NS1 antibodies are also effective both prophylactically and therapeutically in animal models [66]. A possible im- 
munopathological activity in the humoral response to flavivirus infection is known as antibody-dependent enhancement of infection, which means that when antibody-virus complexes are internalized into cells via $\mathrm{FC} \gamma \mathrm{Rs}$, flavivirus replication is enhanced in macrophages [66]. $\mathrm{CD} 8^{+} \mathrm{T}$ cells are particularly important for virus elimination through cytokine- and lysis-dependent mechanisms, while the studies focusing on the strongly induced $\mathrm{CD} 4^{+} \mathrm{T}$ cells are less extensive $[59,66,67]$. Dissecting and understanding the antigenicity and immunity during infection of other flaviviruses could help to elucidate possible approaches to abrogate or ameliorate the disease caused by DEDSV.

\section{Public health}

Flaviviruses are notorious for their severe infective and zoonotic properties. Notably, the flaviviruses, including TMUV [33,34] and BAGV [5,68], that cross-react with DEDSV are all infectious to human beings. Given the close phylogenetic relationship with YFV, DENV, TBEV, and JVE group viruses, as well as the same CS pattern with JEV group viruses [15], DEDSV has high potential to be a zoonotic pathogen. Antibodies against TMUV have been identified in human beings $[33,34]$, and thus DEDSV may have already infected humans with asymptomatic results. Furthermore, the host range of DEDSV has been extended from ducks to other domestic birds such as chickens, geese, pigeons, and even house sparrows. In the future, we hypothesize that DEDSV will adapt to broader hosts, including humans. Similarly, these birds may bring DEDSV to distant regions. With regard to increased and extensive transport activities and global warming, DEDSV can spread more quickly and broadly and continuously evolve. Therefore, the possibility of epidemics of reemerging diseases caused by DEDSV cannot be ignored.

To prevent and control DEDSV, many groups have established efficient assays for the rapid detection of DEDSV. In summary, two primary principals have been employed. The first is a conventional SYBR Green-I-based real-time RT-PCR, which amplifies a 400-bp segment of the E gene [69]. The other is a reverse-transcription loop-mediated isothermal amplification assay (RT-LAMP) [28,69-71]. LAMP, created by Notomi et al. [72], is a rapid, simple, and field-adaptable technique. LAMP primers are designed to recognize 4-6 distinct regions of a target gene, and utilize the principal of a strand displacement reaction, while the amplification reaction occurs at isothermal conditions. The typical features of this method include high specificity and high amplification efficiency. Moreover, it is economical and, most importantly, time saving. Both RT-LAMP and real-time RT-PCR can be performed within 30 min [69]. RT-LAMP is more convenient and economical than realtime RT-PCR, and thus, it is currently the most appropriate choice for the rapid detection of DEDSV.
In the absence of specific DEDS therapeutics, the most feasible strategy to control DEDSV is vaccination. To date, there is still not a licensed DEDSV vaccine yet available. Here, we simply summarize some studies on representative flavivirus vaccines to facilitate future work on DEDSV vaccines.

Many traditional and novel approaches have been employed to develop flavivirus vaccines [73]. Traditional approaches include inactivated vaccines and live attenuated vaccines. However, vaccines against only three flaviviruses so far, i.e., YFV, JEV, and TBEV, are available for human beings [73]. The YFV vaccine, YF-17D is an attenuated YFV strain by multiple passages in animal tissues [74]. JEV vaccine is a formalin inactivated whole virus prepared from mouse brains [73]. The first licensed TBEV vaccine is also a whole inactivated virus vaccine. A common problem of inactivated vaccines is the poor immunogenicity compared to live-attenuated vaccines. Hence, the concept of using live-attenuated vaccines is more and more preferred [75]. The application of modern techniques to vaccine development is opening up new avenues of approach [73]. Novel methods for delivery of live vaccines, such as inoculation of infectious DNA or RNA, construction of protein subunit, expression vector-based and naked DNA vaccines have been proposed to create alternate vaccine candidates [76]. To date, no single approach has proved successful in leading to vaccine development against a wide range of flaviviruses. All of the above work should be valuable for DEDSV vaccine development and we may start with a live attenuated DEDSV strain.

\section{Conclusion and perspectives}

A new flavivirus causing severe diseases to the domestic poultry has been identified in 2010 in China and it has been quickly characterized in detail. Although it is closely related to TMUV and STWV, we proposed a new name as DEDSV based on a comprehensive analysis and its unique geographic distribution and host ranges. Due to the zoonotic nature of its relatives, we also propose for a close surveillance of the virus to scrutinize any potential public health concerns. Vaccines and therapeutic measures should be vigorously pursued to prevent future outbreaks.

Work in Gao George Fu's laboratory is, in part, supported by the National Basic Research Program of China, Ministry of Science and Technology of China (2011CB504703) and National Natural Science Foundation of China (81021003). Gao George Fu is a leading principal investigator of the NSFC Innovative Research Group.

1 Porterfield J S. Antigenic characteristics and classification of togaviridae. In: Schlesinger R W, ed. The Togaviruses. New York: Academic Press, 1980. 13-46

2 Swayne D E, Beck J R, Smith C S, et al. Fatal encephalitis and myocarditis in young domestic geese (Anser anser domesticus) 
caused by West Nile virus. Emerg Infect Dis, 2001, 7: 751-753

3 Austin R J, Whiting T L, Anderson R A, et al. An outbreak of West Nile virus-associated disease in domestic geese (anser anser domesticus) upon initial introduction to a geographic region, with evidence of bird to bird transmission. Can Vet J, 2004, 45: 117-123

4 Barnard B J, Buys S B, Du Preez J H, et al. Turkey meningoencephalitis in South Africa. Onderstepoort J Vet Res, 1980, 47: 89-94

5 Gould E A, Solomon T. Pathogenic flaviviruses. Lancet, 2008, 371: 500-509

6 Kuno G, Chang G J. Biological transmission of arboviruses: reexamination of and new insights into components, mechanisms, and unique traits as well as their evolutionary trends. Clin Microbiol Rev, 2005, 18: 608-637

7 Weaver S C, Barrett A D. Transmission cycles, host range, evolution and emergence of arboviral disease. Nat Rev Microbiol, 2004, 2: 789-801

8 Weissenbock H, Hubalek Z, Bakonyi T, et al. Zoonotic mosquitoborne flaviviruses: worldwide presence of agents with proven pathogenicity and potential candidates of future emerging diseases. Vet Microbiol, 2010, 140: 271-280

9 Komar N. West Nile virus: epidemiology and ecology in North America. Adv Virus Res, 2003, 61: 185-234

10 Guy J S, Malkinson M. Diseases of poultry. In: Saif I M, ed. Arbovirus Infections. Ames: Blackwell Publishing, 2008. 414-425

11 Aguero M, Fernandez-Pinero J, Buitrago D, et al. Bagaza virus in partridges and pheasants, Spain, 2010. Emerg Infect Dis, 2011, 17: 1498-1501

$12 \mathrm{Su} \mathrm{J}$, Li S, Hu X, et al. Duck egg-drop syndrome caused by BYD virus, a new Tembusu-related flavivirus. PLoS ONE, 2011, 6: e18106

13 Yan P, Zhao Y, Zhang X, et al. An infectious disease of ducks caused by a newly emerged Tembusu virus strain in mainland China. Virology, 2011, 417: 1-8

14 Cao Z, Zhang C, Liu Y, et al. Tembusu virus in ducks, China. Emerg Infect Dis, 2011, 17: 1873-1875

15 Liu P, Lu H, Li S, et al. Genomic and antigenic characterization of the newly emerging Chinese duck egg-drop syndrome flavivirus: genomic comparison with Tembusu and Sitiawan viruses. J Gen Virol, 2012, 93: 2158-2170

16 Zhu W, Chen J, Wei C, et al. Complete genome sequence of duck Tembusu virus, isolated from Muscovy ducks in southern China. J Virol, 2012, 86: 13119

17 Yun T, Zhang D, Ma X, et al. Complete genome sequence of a novel flavivirus, duck Tembusu virus, isolated from ducks and geese in China. J Virol, 2012, 86: 3406-3407

18 Wan C, Huang Y, Fu G, et al. Complete genome sequence of avian Tembusu-related virus strain WR isolated from White Kaiya ducks in Fujian, China. J Virol, 2012, 86: 10912

19 Tang Y, Diao Y, Gao X, et al. Analysis of the complete genome of Tembusu virus, a flavivirus isolated from ducks in China. Transbound Emerg Dis, 2012, 59: 336-343

20 Tang Y, Diao Y, Yu C, et al. Characterization of a Tembusu virus isolated from naturally infected house sparrows (Passer domesticus) in Northern China. Transbound Emerg Dis, 2013, 60: 152-158

21 Liu M, Chen S, Chen Y, et al. Adapted Tembusu-like virus in chickens and geese in China. J Clin Microbiol, 2012, 50: 2807-2809

22 Li S, Zhang L, Wang Y, et al. An infectious full-length CDNA clone of duck Tembusu virus, a newly emerging flavivirus causing duck egg drop syndrome in China. Virus Res, 2013, 171: 238-241

23 Li L L, An H J, Sun M H, et al. Identification and genomic analysis of two duck-origin Tembusu virus strains in southern China. Virus Genes, 2012, 45: 105-112

24 Vaidya N K, Wang F B, Zou X, et al. Transmission dynamics of the recently-identified BYD virus causing duck egg-drop syndrome. PLoS ONE, 2012, 7: e35161

25 Yun $\mathrm{T}$, Ye W, Ni $\mathrm{Z}$, et al. Identification and molecular characterization of a novel flavivirus isolated from Pekin ducklings in China. Vet Microbiol, 2012, 157: 311-319

26 Luo L, Wen G, Yang J, et al. Histopathological observation of ducks infected naturally with duck flavivirus. Prog Vet Med, 2012, 33: 4

27 Huang $\mathrm{X}$, Han $\mathrm{K}$, Zhao D, et al. Identification and molecular characterization of a novel flavivirus isolated from geese in China. Res Vet Sci, 2013, 94: 774-780

28 Yan L, Yan P, Zhou J, et al. Establishing a taqman-based real-time PCR assay for the rapid detection and quantification of the newly emerged duck Tembusu virus. Virol J, 2011, 8: 464

29 Peleg J. Behaviour of infectious RNA from four different viruses in continuously subcultured Aedes aegypti mosquito embryo cells. Nature, 1969, 221: 193-194

30 Liu M, Liu C, Li G, et al. Complete genomic sequence of duck flavivirus from China. J Virol, 2012, 86: 3398-3399

31 Pletnev A, Gould E A, Heinz F X, et al. Flaviviridae. In: Andrew M Q K, Michael J A, Eric B C, et al., eds. Virus Toxonomy. Oxford: Elsevier, 2011. 1003-1020

32 Institute for Medical Research FoM. Annual report. 1957, 100-103

33 Wolfe N D, Kilbourn A M, Karesh W B, et al. Sylvatic transmission of arboviruses among Bornean orangutans. Amer J Trop Med Hyg, 2001, 64: 310-316

34 Mackenzie J S, Williams D T. The zoonotic flaviviruses of southern, south-eastern and eastern Asia, and Australasia: the potential for emergent viruses. Zoonoses Public HLTH, 2009, 56: 338-356

35 Gould E A, de Lamballerie X, Zanotto P M A, et al. Origins, evolution, and vector/host coadaptations within the Genus Flavivirus. 2003, 59: 277-314

36 Gaunt M W, Sall A A, Lamballerie X, et al. Phylogenetic relationships of flaviviruses correlate with their epidemiology, disease association and biogeography. J Gen Virol, 2001, 82: 1867-1876

37 Thurner C, Witwer C, Hofacker I L, et al. Conserved RNA secondary structures in Flaviviridae genomes. J Gen Virol, 2004, 85: 1113-1124

38 Gritsun T S, Gould E A. Origin and evolution of flavivirus 5' UTRs and panhandles: trans-terminal duplications? Virology, 2007, 366: $8-15$

39 Gritsun T S, Gould E A. Direct repeats in the flavivirus 3' untranslated region; a strategy for survival in the environment? Virology, 2007, 358: 258-265

40 Gritsun T S, Gould E A. Origin and evolution of 3'UTR of flaviviruses: long direct repeats as a basis for the formation of secondary structures and their significance for virus transmission. Adv Virus Res, 2006, 69: 203-248

41 Villordo S M, Gamarnik A V. Genome cyclization as strategy for flavivirus RNA replication. Virus Res, 2009, 139: 230-239

42 Yu L, Nomaguchi M, Padmanabhan R, et al. Specific requirements for elements of the $5^{\prime}$ and $3^{\prime}$ terminal regions in flavivirus RNA synthesis and viral replication. Virology, 2008, 374: 170-185

43 Alvarez D E, Lodeiro M F, Luduena S J, et al. Long-range RNA-RNA interactions circularize the dengue virus genome. J Virol, 2005, 79: 6631-6643

44 Khromykh A A, Meka H, Guyatt K J, et al. Essential role of cyclization sequences in flavivirus RNA replication. J Virol, 2001, 75: 6719-6728

45 Filomatori C V, Lodeiro M F, Alvarez D E, et al. A 5' RNA element promotes dengue virus RNA synthesis on a circular genome. Genes Dev, 2006, 20: 2238-2249

46 Wei Y, Qin C, Jiang T, et al. Translational regulation by the $3^{\prime}$ untranslated region of the dengue type 2 virus genome. Am J Trop Med Hyg, 2009, 81: 817-824

47 Friebe P, Harris E. Interplay of RNA elements in the dengue virus 5' and $3^{\prime}$ ends required for viral RNA replication. J Virol, 2010, 84: 6103-6118

48 Friebe P, Shi P Y, Harris E. The 5' and $3^{\prime}$ downstream AUG region elements are required for mosquito-borne flavivirus RNA replication. J Virol, 2011, 85: 1900-1905

49 Lodeiro M F, Filomatori C V, et al. Structural and functional studies of the promoter element for dengue virus RNA replication. J Virol, 2009, 83: 993-1008

50 Gebhard L G, Filomatori C V, Gamarnik A V. Functional RNA 
elements in the dengue virus genome. Viruses, 2011, 3: 1739-1756

51 Leyssen P, Charlier N, Lemey P, et al. Complete genome sequence, taxonomic assignment, and comparative analysis of the untranslated regions of the Modoc virus, a flavivirus with no known vector. Virology, 2002, 293: 125-140

52 Clyde K, Harris E. RNA secondary structure in the coding region of dengue virus type 2 directs translation start codon selection and is required for viral replication. J Virol, 2006, 80: 2170-2182

53 Zuker M. Mfold web server for nucleic acid folding and hybridization prediction. Nucleic Acids Res, 2003, 31: 3406-3415

54 Brinton M A, Fernandez A V, Dispoto J H. The 3'-nucleotides of flavivirus genomic RNA form a conserved secondary structure. Virology, 1986, 153: 113-121

55 Hahn C S, Hahn Y S, Rice C M, et al. Conserved elements in the $3^{\prime}$ untranslated region of flavivirus RNAs and potential cyclization sequences. J Mol Biol, 1987, 198: 33-41

56 Vazquez-Calvo A, Saiz J C, McCullough K C, et al. Acid-dependent viral entry. Virus Res, 2012, 167: 125-137

57 Lee E, Stocks C E, Amberg S M, et al. Mutagenesis of the signal sequence of yellow fever virus prm protein: enhancement of signalase cleavage in vitro is lethal for virus production. J Virol, 2000, 74: 24-32

58 Lindenbach B D, Thiel H J, Rice C M. Flaviviridae: the viruses and their replication. In: David M K, Peter M H, eds. Fields Virology. Philadelphia: Lippincott Williams \& Wilkins, 2007. 1102-1153

59 Pastorino B, Nougairede A, Wurtz N, et al. Role of host cell factors in flavivirus infection: implications for pathogenesis and development of antiviral drugs. Antiviral Res, 2010, 87: 281-294

60 Gubler D J, Kuno G, Markoff L. Flaviviruses. In: David M K, Peter M H, eds. Fields Virology. Philadelphia: Lippincott Williams \& Wilkins, 2007. 1154-1252

61 Luca V C, AbiMansour J, Nelson C A, et al. Crystal structure of the Japanese encephalitis virus envelope protein. J Virol, 2012, 86: 2337-2346

62 Nybakken G E, Nelson C A, Chen B R, et al. Crystal structure of the West Nile virus envelope glycoprotein. J Virol, 2006, 80: $11467-11474$
63 Modis Y, Ogata S, Clements D, et al. A ligand-binding pocket in the Dengue virus envelope glycoprotein. 2003, 100: 6986-6991

64 Rey F A, Heinz F X, Mandl C, et al. The envelope glycoprotein from tick-borne encephalitis virus at $2 \AA$ resolution. Nature, 1995, 375: 291-298

65 Roehrig J T. Antigenic structure of flavivirus proteins. Adv Virus Res, 2003, 59: 141-175

66 King N J, Getts D R, Getts M T, et al. Immunopathology of flavivirus infections. Immunol Cell Biol, 2007, 85: 33-42

67 Sips G J, Wilschut J, Smit J M. Neuroinvasive flavivirus infections. Rev Med Virol, 2012, 22: 69-87

68 Bondre V P, Sapkal G N, Yergolkar P N, et al. Genetic characterization of Bagaza virus (BAGV) isolated in India and evidence of anti-BAGV antibodies in sera collected from encephalitis patients. J Gen Virol, 2009, 90: 2644-2649

69 Jiang T, Liu J, Deng Y Q, et al. Development of RT-LAMP and realtime RT-PCR assays for the rapid detection of the new duck Tembusu-like BYD virus. Arch Virol, 2012, 157: 2273-2280

70 Wang Y, Yuan X, Li Y, et al. Rapid detection of newly isolated Tembusu-related flavivirus by reverse-transcription loop-mediated isothermal amplification assay. Virol J, 2011, 8: 553

71 Tang Y, Diao Y, Yu C, et al. Rapid detection of Tembusu virus by reverse-transcription, loop-mediated isothermal amplification (RTLAMP). Transbound Emerg Dis, 2012, 59: 208-213

72 Notomi T, Okayama H, Masubuchi H, et al. Loop-mediated isothermal amplification of DNA. Nucleic Acids Res, 2000, 28: E63

73 Pulmanausahakul R, Khakpoor A, Smith D R. The development of flavivirus vaccines. Afr J Biotechnol, 2010, 9: 409-415

74 Theiler M, Smith H H. The use of yellow fever virus modified by in vitro cultivation for human immunization. J Exp Med, 1937, 65: 787-800

75 Koraka P, Martina B E E, Osterhaus A. Bioinformatics in new generation flavivirus vaccines. J Biomed Biotechnol, 2010, 2010: 864029

76 Pugachev K V, Guirakhoo F, Trent D W, et al. Traditional and novel approaches to flavivirus vaccines. Int J Parasitol, 2003, 33: 567-582

Open Access This article is distributed under the terms of the Creative Commons Attribution License which permits any use, distribution, and reproduction in any medium, provided the original author(s) and source are credited. 